\title{
Fock space localization, return probability, and conductance of disordered interacting electrons
}

\author{
Frank Epperlein, Svetlana Kilina, Michael Schreiber, Sergey Uldanov, and Thomas Vojta ${ }^{1}$ \\ Institut für Physik, Technische Universität Chemnitz, D-09107 Chemnitz, Germany
}

\begin{abstract}
We numerically simulate the low-energy properties of interacting electrons in a random potential using the HartreeFock based exact diagonalization method. In particular, we investigate how the transport properties are influenced by the combined effects of disorder and correlations in the presence of the electron spin. To this end we calculate the participation number of many-particle states in Fock space, the return probability of single-particle excitations, and the Kubo-Greenwood conductance. It turns out that in the strongly localized regime interactions increase the conductance whereas for weak disorder interactions decrease the conductance. In contrast, single-particle excitations in general experience a localizing influence of the interactions.
\end{abstract}

Key words: electronic transport, disorder, localization, metal-insulator transitions

\section{Introduction}

The interplay between Anderson localization and electron-electron interactions belongs to the most important unsolved problems of today's condensed matter physics. The standard theoretical approach to this problem is based on perturbation theory in both disorder and interactions. In the metallic regime, the interactions lead to quantum corrections to the conductivity in addition to the weak-localization corrections [1]. A scaling theory $[2,3]$ was developed on the basis of these perturbative results. It predicts that a phase transition between an insulator and a normal metal, i.e., a disordered Fermi liquid, is possible only for

1 Corresponding author.

E-mail: vojta@physik.tu-chemnitz.de dimensions $d>2$. In $d=2$ the situation is inconclusive. Under renormalization the disorder flows to zero but the interaction strength diverges, i.e., the perturbation theory becomes uncontrolled.

The attention to these questions resurged in the mid nineties, mainly due to two surprising developments. First, Shepelyansky suggested [4] that in the localized regime two interacting particles can form a pair whose localization length is much larger than that of a single particle. Second, measurements [5] on high-quality Si-MOSFETs revealed indications of a true metal-insulator transition (MIT) in two dimensions. Since these experiments are carried out at low electron density where the Coulomb interaction is particularly strong compared to the Fermi energy, interaction effects are a likely reason for this phenomenon. A 
complete understanding has, however, not yet been obtained. In a number of theories new quantum states of matter have been postulated, either nonFermi liquid metals or unusual superconductors [6-8]. However, other explanations based on more conventional physics like temperature-dependent disorder [9] or impurity screening [10], imply that the seeming MIT is a transient phenomenon, and the true ground state is always an insulator.

We have recently developed $[11,12]$ an efficient method to investigate disordered interacting electrons numerically, the Hartree-Fock (HF) based exact diagonalization (HFD). It is related to the quantum-chemical configuration interaction approach. We have used this method to study the influence of interactions on the conductance of spinless fermions in one, two, and three dimensions. We found a delocalizing tendency of the interactions for strong disorder but a localizing one for weak disorder. In this work we extend our HFD investigations to electrons with spin. In particular, we calculate the participation number of the many-particle eigenstates in Fock space, the localization properties of single-particle excitations, and the Kubo-Greenwood conductance.

\section{Model and method}

The generic model for spinless interacting disordered electrons is the quantum Coulomb glass $[13,14]$. In this paper we use a generalization of the quantum Coulomb glass to electrons with spin. It is defined on a regular square lattice with $\mathcal{N}=L^{2}$ sites occupied by $N=N_{\uparrow}+N_{\downarrow}=2 K \mathcal{N}$ electrons $(0<K<1)$. To ensure charge neutrality each lattice site carries a compensating positive charge of $2 \mathrm{Ke}$. The Hamiltonian is given by

$$
\begin{aligned}
H= & -t \sum_{\langle i j\rangle, \sigma}\left(c_{i \sigma}^{\dagger} c_{j \sigma}+\text { h.c. }\right)+\sum_{i, \sigma} \varphi_{i} n_{i \sigma} \\
& +\frac{1}{2} \sum_{i \neq j, \sigma, \sigma^{\prime}} U_{i j}\left(n_{i \sigma}-K\right)\left(n_{j \sigma^{\prime}}-K\right) \\
& +U_{H} \sum_{i} n_{i \uparrow} n_{i \downarrow}
\end{aligned}
$$

where $c_{i \sigma}^{\dagger}, c_{i \sigma}$, and $n_{i \sigma}$ are the creation, annihilation and occupation number operators at site $i$ and spin $\sigma .\langle i j\rangle$ denotes all pairs of nearest-neighbor sites. $t$ is the strength of the hopping term, i.e., the kinetic energy. We parametrize the interaction $U_{i j}=e^{2} / r_{i j}$ by its value $U$ between nearestneighbor sites and include a Hubbard interaction $U_{H}$. The random potential values $\varphi_{i}$ are chosen independently from a box distribution of width $2 W_{0}$ and zero mean. The boundary conditions are periodic and the Coulomb interaction is treated in the minimum image convention (which implies a cutoff at a distance of $L / 2$ ).

A numerically exact solution of a quantum many-particle system requires the diagonalization of a matrix whose dimension increases exponentially with system size. This severely limits the possible sizes. To overcome this problem we have developed the HFD method. The basic idea is to work in a truncated Hilbert space consisting of the HF ground state and the low-lying excited Slater states. For each disorder configuration three steps are performed: (i) find the HF solution of the problem, (ii) determine the $B$ Slater states with the lowest energies, and (iii) calculate and diagonalize the Hamilton matrix in the subspace spanned by these states. The number $B$ of basis states determines the quality of the approximation, reasonable values have to be found empirically.

\section{Results}

To characterize, to what extent the interactions introduce non-trivial correlations (beyond the HF level) into the system we calculate the participation number of the many-particle ground state with respect to the HF Slater determinants. The results for a system of $4 \times 4$ sites at half filling are shown in Fig. 1. For $U=0$ the Fock space participation number is 1 because the HF approximation is (trivially) exact for non-interacting electrons. Increasing the interaction mixes the single-particle states, and the inverse Fock space participation 


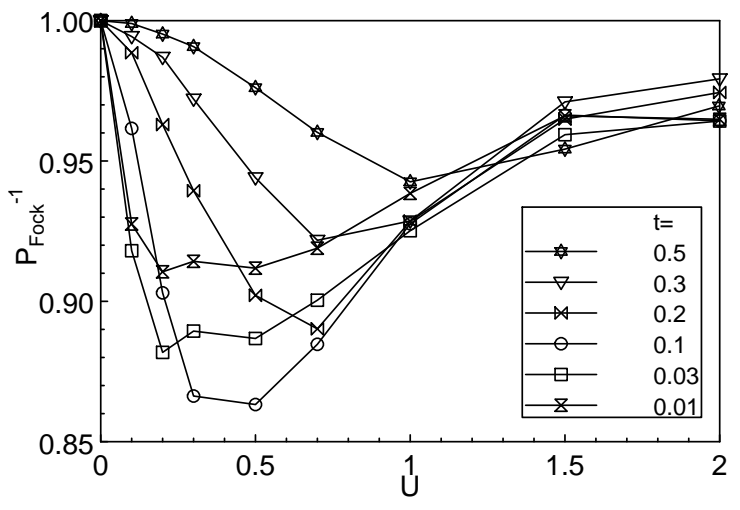

Fig. 1. Inverse Fock space participation number $P_{\text {Fock }}^{-1}$ as a function of interaction $U$ for a system of $4 \times 4$ sites occupied by 8 spin-up and 8 spin-down electrons. The disorder is $W_{0}=1$, the Hubbard energy $U_{H}=U$, and the HFD basis size $B=500$. The data represent averages over 1000 disorder configurations.

number is reduced. Nontrivial interaction effects, if any, become manifest mostly in this region. For even larger interactions the eigenstates become again more localized in Fock space since the HF approximation becomes exact again for $U \rightarrow \infty$.

Analogous information can be extracted from the single-particle survival probability

$$
Z(\omega)=\frac{1}{N} \sum_{i j} \lim _{\delta \rightarrow 0} \frac{\delta}{\pi} G_{i j}^{R}(\omega+i \delta) G_{j i}^{A}(\omega-i \delta),
$$

which averages the sum of the transmission probabilities of a single-particle excitation to all sites. Here $G_{i j}^{R, A}(\omega)$ are the retarded and advanced single-particle Greens functions. The survival probability at the Fermi energy, shown in Fig. 2, is related to the square of the quasiparticle weight.

We now turn to the single-particle localization properties which we characterize by the return probability of the single-particle excitations,

$R_{p}(\omega)=\frac{1}{N} \sum_{i} \lim _{\delta \rightarrow 0} \frac{\delta}{\pi} G_{i i}^{R}(\omega+i \delta) G_{i i}^{A}(\omega-i \delta) .(3)$

However, in this quantity localization and decay of the quasiparticles are entangled. To extract the localization properties we normalize $R_{p}$ by the survival probability $Z$. The data in Fig. 3 show that, in general, interactions tend to localize the singleparticle excitations. This is in agreement with ear-

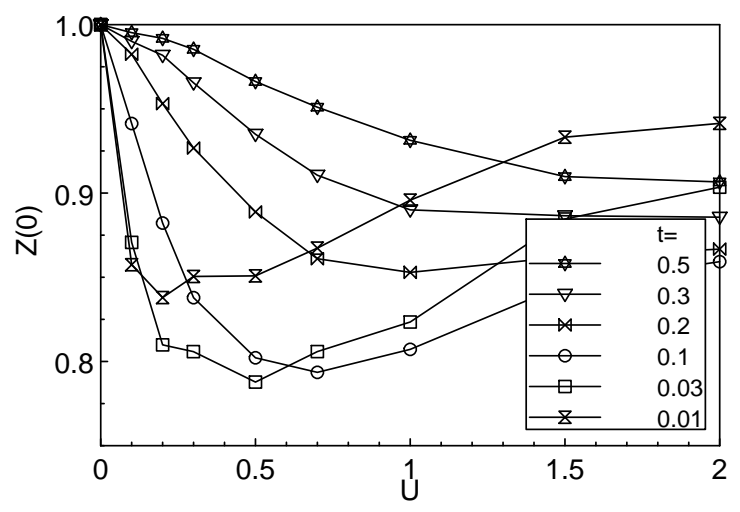

Fig. 2. Single-particle survival probability at the Fermi energy, $Z(0)$; parameters as in Fig. 1.

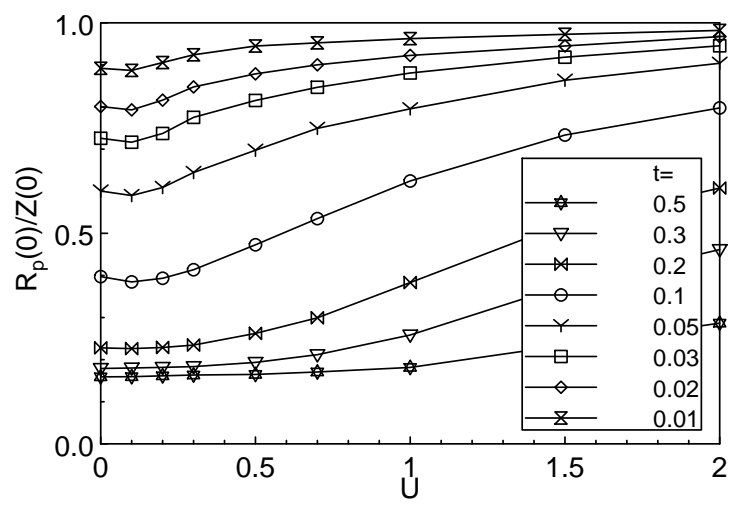

Fig. 3. Normalized single-particle return probability at the Fermi energy, $R_{p}(0) / Z(0)$; parameters as in Fig. 1.

lier HF results [14] which showed that the Coulomb gap in the single-particle density of states is responsible for the trend towards localization. In Fig. 3 a tiny delocalization seems to occur at low interaction strength and small kinetic energy. However, the changes are within the statistical errors of our study.

In a real transport experiment the most accessible observable is the conductance. Theoretically, it can be obtained from linear-response theory. It is essentially determined by the current-current correlation function of the ground state. The real (dissipative) part of the conductance (in units of $e^{2} / h$ ) at frequency $\omega$ is given by the Kubo-Greenwood formula [15], 


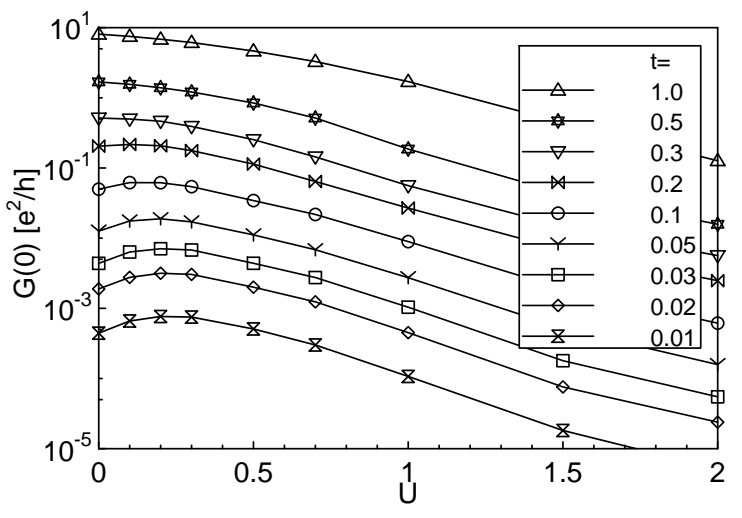

Fig. 4. d.c. conductance $G(0)$ for the same system as in Fig. 1. The inhomogeneous broadening is $\gamma=0.0625$.

$\operatorname{Re} G^{x x}(\omega)=\frac{2 \pi^{2}}{\omega} \sum_{\nu}\left|\left\langle 0\left|j^{x}\right| \nu\right\rangle\right|^{2} \delta\left(\omega+E_{0}-E_{\nu}\right)$.(4)

$j^{x}$ is the $x$ component of the current operator and $|\nu\rangle$ is an eigenstate of the Hamiltonian. Eq. (4) describes an isolated system while in a real d.c. transport experiment the sample is connected to contacts and leads. This results in a finite life time $\tau$ of the eigenstates leading to an inhomogeneous broadening $\gamma=\tau^{-1}$ of the $\delta$ functions in (4) [16]. To suppress the discreteness of the spectrum of a finite system, $\gamma$ should be at least of the order of the single-particle level spacing. Here, this requires comparatively large $\gamma \geq 0.05$. We tested different $\gamma$ and found that the conductance values depend on $\gamma$ but the qualitative results do not.

The typical d.c. conductance is shown in Fig. 4. Since the logarithm of the conductance rather than the conductance itself is a self-averaging quantity in a disordered system we calculate the typical conductance by averaging the logarithms of the conductance values of 1000 disorder configurations. We find that, as in the spinless case, there are two qualitatively different regimes. For large kinetic energy $t$ the interactions always reduce the d.c. conductance, while for small kinetic energy, i.e. in the localized regime, small and moderate interactions significantly enhance the d.c. conductance. For larger interaction strength the conductance drops, indicating the crossover to a Wigner crystal or Wigner glass.
A comparison with analogous results for spinless fermions [11] shows that the delocalizing influence of the interactions is significantly stronger for electrons with spin.

This work was supported in part by the DFG under Grant No. SFB393/C2 and by the NSF under Grant No. DMR-98-70597.

\section{References}

[1] See, e.g., B. L. Altshuler and A. G. Aronov, in A. L. Efros and M. Pollak (Eds.) Electron-electron interactions in disordered systems, North-Holland, Amsterdam (1985).

[2] A. M. Finkelstein, Zh. Eksp. Teor. Fiz. 84, 168 (1983) [Sov. Phys. JETP 57, 97 (1983)].

[3] D. Belitz and T. R. Kirkpatrick, Rev. Mod. Phys. 66, 261 (1994).

[4] D. L. Shepelyansky, Phys. Rev. Lett. 73, 2607 (1994).

[5] S. V. Kravchenko et al., Phys. Rev. Lett. 77, 4938 (1996).

[6] C. Castellani, C. DiCastro and P. A. Lee, Phys. Rev. B 57, R9381 (1998).

[7] Q. Si and C. M. Varma, Phys. Rev. Lett. 81, 4951 (1998); S. Chakravarty et al., Phil. Mag. B 79, 859 (1999); G. Benenti, X. Waintal, and J.-L. Pichard, Phys. Rev. Lett. 83, 1826 (1999).

[8] F. C. Zhang and T. M. Rice, cond-mat/9708050; D. Belitz and T. R. Kirkpatrick, Phys. Rev. B 58, 8214 (1998); P. Phillips et al., Nature 395, 253 (1998).

[9] B. L. Altshuler and D. L. Maslov, Phys. Rev. Lett. 82 145 (1999).

[10] A. Gold and V. T. Dolgopolov, Phys. Rev. B 33, 1076 (1986); S. Das Sarma and E. H. Hwang, Phys. Rev. Lett. 83, 164 (1999).

[11] T. Vojta, F. Epperlein, and M. Schreiber, Phys. Rev. Lett. 81, 4212 (1998).

[12] T. Vojta, F. Epperlein, and M. Schreiber, Computer Phys. Commun. 121-122, 489 (1999).

[13] A. L. Efros and F. G. Pikus, Solid State Commun. 96, 183 (1995); J. Talamantes, M. Pollak, and L. Elam, Europhys. Lett. 35, 511 (1996).

[14] F. Epperlein, M. Schreiber, and T. Vojta, Phys. Rev. B 56, 5890 (1997).

[15] K. Kubo, J. Phys. Soc. Jpn. 12, 576 (1957); D. A. Greenwood, Proc. Phys. Soc. 71, 585 (1958).

[16] See, e.g., S. Datta, Electronic transport in mesoscopic systems, Cambridge University Press, Cambridge (1997). 